\title{
ZBTB7A suppresses castration-resistant prostate cancer
}

Possible mechanisms underlying the development of castration-resistant prostate cancer have been identified, with a key culprit being the gene $Z B T B 7 A$. $Z B T B 7 A$ is a known transcription factor that has been shown to be highly expressed in non-Hodgkin lymphoma, repressing the tumour-suppressor gene $A R F$. In this context, as well as in non-small-cell lung, breast and ovarian cancers, ZBTB7A acts as a proto-oncogene. To explore the role of this gene in prostate cancer, Pier Paolo Pandolfi and his team of researchers generated a transgenic mouse model that overexpresses $Z B T B 7 A$ in the prostate. To their surprise, the overexpression failed to result in neoplastic transformation.

Instead, highly penetrant adenocarcinomas were observed in mice with inactivated $Z B T B 7 A$ and $P T E N$, loss of which is a known driver of prostate cancer. In fact, $Z B T B 7 A$ inactivation accelerated tumorigenesis driven by PTEN loss. Furthermore, the ZBTB7A protein interacts with Sox9 to antagonize its transcriptional activity on genes such as MIA, which is involved in tumour cell invasion. Thus, ZBTB7A is oncosuppressive in PTEN-null tumours of the prostate.

\section{4 ...stratification of patients by ZBTB7A and PTEN status might be informative 77}

The researchers then went on to explore the clinical implications of these findings, and showed that castration of PTEN-null mice led to the induction of apoptosis and proliferation blockade and initial tumour shrinkage. However, this favourable response was bypassed in PTEN-null, ZBTB7A-null animals, leading to the development of castration resistance.

Analysis of samples from patients with prostate cancer revealed that men whose tumours had high levels of ZBTB7A-null cells responded poorly to androgen-deprivation therapy (ADT).
By contrast, PTEN loss alone could not differentiate poor responders from favourable responders, which suggests that stratification of patients by ZBTB7A and PTEN status might be informative.

Furthermore, the mechanism of ZBTB7A loss in triggering resistance to ADT might be mediated via the downregulation of $X A F 1$ and upregulation of SRD5A1, a pattern that was

demonstrated in the mouse and human tissue studies. These biomarkers might be valuable diagnostic and prognostic tools as well as therapeutic targets-indeed, therapy targeting this mechanism in mouse models was effective in vivo.

Mina Razzak

Original articles Wang, G. et al. Zbtb7a suppresses prostate cancer through repression of a Sox9-dependent pathway for cellular senescence bypass and tumor invasion. Nat. Genet. doi:10.1038/ng.2654 | Lunardi, A. et al. A co-clinical approach identifies mechanism and potential therapies for androgen deprivation resistance in prostate cancer. Nat. Genet. doi:10.1038/ng.2650 